\title{
Redefining Qualitative Benchmarks of Theories and Models: An Empirical Exploration of Fast and Slow Errors in Speeded Decision-making
}

\author{
Gabriel Tillman ${ }^{a, b}$ and Nathan J. Evans ${ }^{c, d}$ \\ ${ }^{a}$ School of Science, Psychology and Sport, Federation University, Australia \\ ${ }^{b}$ Discipline of Psychology, Australian College of Applied Psychology, Australia \\ ${ }^{c}$ School of Psychology, University of Queensland, Australia \\ ${ }^{d}$ Department of Psychology, University of Amsterdam, The Netherlands
}

Word Count: 4301

Author Note

Correspondence concerning this article should be addressed to Gabriel Tillman, School of Health and Life Sciences, Federation University, Australia

Contact: gabrieltillman.au@gmail.com

NJE was supported by an Australian Research Council Discovery Early Career Researcher Award (DE200101130) and a European Research Council Advanced Grant (743086 UNIFY). 


\begin{abstract}
Qualitative benchmarks in empirical data constrain the theories and models researchers generate about any given phenomena. In the field of decision-making, two key qualitative benchmarks are fast and slow errors. To account for these benchmarks researchers have added two complicated between-trial variability mechanisms to prominent decision making models, but the reliability of these benchmarks is yet to be determined. Our study aimed to provide an assessment of the reliability of fast and slow errors at both the group and individual subject level. We used archival and newly collected data and analysed these data under Bayesian frameworks. We found inconsistencies in the benchmarks between experiments and between subjects within the same experiment. Our work not only questions the reliability of fast and slow errors, but also the minimum requirements for a pattern of data to be classed as a qualitative benchmark that forms the basis for psychological theory.
\end{abstract}

Keywords: Speeded Decision Making, Diffusion Model, Qualitative Benchmarks 
Decision-making is a process that takes time, and at least since Donders (1868) researchers have used the time taken to make decisions as a window into the human mind. The time taken to make a decision in combination with the choice that is made are two characteristics that constrain our theories and models of speeded decision making. Speeded decisions may involve something like deciding whether a cloud of dots is moving to the left or right or whether a patch of pixels is mostly dark or mostly bright. Although such decisions seem trivial they are the foundation of our perceptual experience.

Further exploration into performance in speeded decision making tasks revealed that there are nuanced trends in the data. Response time (RT) distributions are right skewed despite many researchers treating the data as normal. The relative speed of correct and error responses are not equal (Laming, 1968; Ratcliff, 1978; Ratcliff \& Rouder, 1998; Tillman, Van Zandt, \& Logan, 2020), where the error RT is either faster than the correct RT on average (i.e., fast errors) or slower than the correct RT on average (i.e., slow errors). Slow errors occur when the choice is difficult or accuracy is emphasized and fast errors occur when the choice is easy or there is pressure to decide quickly (Swensson, 1972).

These nuanced trends formed the qualitative benchmarks for the most dominant theories of how people make speeded decisions: evidence accumulation (see Donkin \& Brown, 2018, for a review) via sequential sampling models (e.g., Ratcliff, 1978; Usher \& McClelland, 2001; Brown \& Heathcote, 2008). Sequential sampling models assume people continually sample evidence from a stimulus, which accumulates from a starting point at a rate of drift rate towards response thresholds that are associated with specific responses to the stimulus. When the accumulated evidence crosses a response threshold, 
this triggers the corresponding overt response. One prominent sequential sampling model, the diffusion model (Ratcliff, 1978), has been highly successful because of its ability to account for key qualitative benchmarks: the positive skew in both correct and error RT distributions, the corresponding accuracy rates, the speed-accuracy trade-off (e.g., Wickelgren, 1977; Luce, 1986; Heitz, 2014), and the relative speed of correct and error RTs.

While the basic diffusion framework (i.e., the Wiener process; Stone, 1960) can account for most of these benchmarks, the model makes a strong prediction that the relative speed of correct and error responses are equal, making it unable to explain fast and slow errors. To account for fast and slow errors, the full diffusion model includes two additional components (Ratcliff \& Rouder, 1998). The first is between-trial variability in drift rate, which allows the model to predict slow errors (Ratcliff, 1978). The second component is between-trial starting point variability, which allows the model to predict fast errors (Laming, 1968; Smith \& Vickers, 1988; Ratcliff \& Rouder, 1998; Ratcliff, Van Zandt, \& McKoon, 1999).

Tillman et al. (2020) argued that there are several issues that arise when adding in additional sources of variability. First, sequential sampling models have become increasingly complex in order to fit RT data and adding in the variability parameters involves two numerical integrations that can be computationally demanding. Second, the between-trial variability assumptions are not part of the process model that explains how the RT data are generated. Tillman et al. (2020) noted that the inclusion of between-trial variability in starting points or drift rates depended on the existence and magnitude of fast and slow errors. Yet, the exact magnitudes of fast and slow errors are rarely reported by researchers because they are usually not the focus of the 
experiment. Therefore, it is difficult to determine if fast and slow errors, which are a key reason for including complex decision components such as between-trial variability in drift rate and starting point, are ubiquitous effects.

Haaf and Rouder (2017) proposed that individual consistency of experimental effects should be a theoretical gold standard for establishing ubiquitous effects, which would separate these effects from other effects that show inconsistency across people. For instance, this gold standard would require that all participants demonstrate fast errors in an experiment rather than only manifesting fast errors at the group level (i.e., manifesting in the group mean RTs). One benefit of showing that an effect occurs for every participant is that the theories we generate from the data will likely generalise to more people in the population of interest.

Our study aims to provide a more definitive assessment of slow and fast errors in decision-making tasks, using standard methodology and the methodology of Haaf and Rouder (2017) to determine if these effects consistently occur at the group-level and the individual subject level, respectively. Our paper will provide evidence for the absence or existence of fast and slow error effects via Bayes factors, and where effects exist, we will provide posterior distributions of the effect sizes. We use archival and newly collected experimental data that use speed-accuracy emphasis manipulations. In what follows, we report the 3 data sets used, the group level analyses, the subject level analyses, and then provide a general discussion of our results.

\section{Data Sets}

\section{Data Set 1}

For data set 1, we used an experiment from Rae, Heathcote, Donkin, Averell, and Brown (2014) that included 49 participants. The data screening process we used was 
identical to Rae et al., removing 15 participants based on inadequate performance. In the task, participants made brightness discriminations of pixel arrays displayed on a computer screen. Specifically, they were asked to determine whether the arrays were of high or low brightness. The authors manipulated speed and accuracy emphasis on alternating blocks of trials. In the accuracy blocks, participants were told to minimise their errors even if their responses become slow. In the speed blocks, participants were told to go quickly and not to worry if they make extra mistakes because the researcher was interested in fast decisions. Participants were given feedback for correct or incorrect responses: CORRECT (in green font) or INCORRECT (in red font), respectively. Finally, if responses were made less than $250 \mathrm{~ms}$, TOO FAST (in red font) was displayed.

\section{Data Set 2}

For data set 2, we used an experiment from Evans, Rae, Bushmakin, Rubin, and Brown (2017) that included 49 participants. The data screening process was identical to Evans et al., removing 2 participants based on inadequate performance. Participants also completed the need for closure scale (Kruglanski, Atash, De Grada, Mannetti, \& Pierro, 2013), which we do not assess here, and is the only key difference between data set 1 and 2 .

\section{Data Set 3}

Data set 3 was a new experiment that resembles both data set 1 and 2 . The complete method of this experiment is presented below.

Participants. The sample comprised of 139 participants made up of members from the general public and first year psychology students at the Australian College of Applied Psychology (ACAP). The age of the participants ranged from 18 to 64 years 
$(\mathrm{M}=33.02, \mathrm{SD}=10.92)$. The sample included 94 participants that identified as female. Participants from the general public were randomly recruited via Facebook advertisement, where they were offered reimbursement for their participation by entering a draw to win 1 of $4 \$ 50$ gift cards. First-year psychology students at ACAP were recruited by signing up through SONA, where they participated for course credit. All participants consented to their participation and indicated that they met the inclusion criteria of the study; over 18 years of age, proficiency in English, and normal or corrected to normal vision.

Material. The stimuli were $64 \times 64$ squares of black and white pixels. Brightness of the square was manipulated by varying the probability that a pixel was white. Four checkerboard patterns, each $64 \times 64$ pixels, were used to mask each stimulus; presented sequentially, they were a checkerboard with $2 \times 2$ black and white squares, a checkerboard the same as the first but with the black and white squares reversed, a checkerboard with $3 \times 3$ black and white squares, and its reverse (see Ratcliff, 2002). Psychopy (Peirce, 2007), an open-source application for online experiments, was used to display stimuli and recording timing and choice data for the brightness discrimination task. Participants used their personal laptop or computer to complete the questionnaire and experiment other devices did not load the experiment.

Procedure. Participants accessed the study via a link which lead them to Qualtrics. This link was accessed through SONA for ACAP first year psychology students, or through the Facebook advertisement for all other participants. Once in Qualtrics, participants were provided with all necessary information about the study including its purpose, the procedure, duration, inclusion criteria, risks and confidentiality. Consent of participation indicated that participants consented to 
participation and that they met the inclusion criteria of the study. Participants first completed the questionnaire which included gathering demographic information (age and gender). After the questionnaire was completed, participants were led to a page which invited them to complete the brightness discrimination task.

There were two levels of brightness, achieved with two values for the probability of a pixel being white: .45 and .55 . These were presented for 150 msec. Each trial began with a plus sign fixation point presented on a grey background for $250 \mathrm{msec}$. Then the stimulus was displayed, followed by the four checkerboard masks displayed for 17 msec each. Then a grey background was presented until a response was made. In accuracy blocks, if a response was correct, there was a 500-msec pause and then the next trial. Feedback was provided in the accuracy conditions. The message "Correct" was displayed in green if they made the correct response, and the message "Try Again" was displayed in red when an incorrect response was made. In the speed emphasis condition, there was no feedback after responses and if a response was longer than 700 ms, the message 'too slow' was displayed. There were 3 blocks of the accuracy condition alternated with three blocks of the speed condition. There were 100 trials per block for a total of 600 trials.

Data Treatment. In line with Ratcliff (1993), long and short outlier RTs were removed. That is, RTs on individual trials that were less than $200 \mathrm{~ms}$ were removed as these responses were considered guesses. RT scores greater than 3 seconds were also removed as these no longer measured perceptual decisions and may have reflected other factors such as lapses in attention 


\section{Group Level Analyses}

In all group level analyses for the three studies in question, we followed almost identical statistical approaches. We used a Bayesian repeated measures ANOVA that included the main effects of Emphasis (Speed and Accuracy), Response (Correct and Error), and an interaction between the two factors. We analyzed the data with JASP (The JASP Team, 2019). We quantified the relative evidence for the null hypothesis and a number of alternative hypotheses using the Bayes factor. The null hypothesis posits that all conditions have identical mean RTs. The key alternative hypothesis we wanted to test was an interaction between Emphasis and Response. Where there were interactions we used simple effects analyses to check for a cross over effect where speed emphasis induces fast errors and accuracy emphasis induces slower errors.

For the ANOVA, we assigned a zero centred Cauchy prior distribution with fixed effects $r=0.5$, random effects $r=1$, and covariates $r=0.354$. Post-hoc comparisons for simple effects were tested using Bayesian t-tests with a zero centred Cauchy prior distribution with $r=1 / \operatorname{sqrt}(2)$. In terms of parameter estimation, we were interested in the posterior distribution of the standardized effect size $\delta$ for the simple effects of the ANOVA, which is the population version of Cohen's d. We estimated $\delta$ for the difference in correct and error mean RT in both the Emphasis conditions. The $\delta$ parameter was assigned a zero centred Cauchy prior distribution with $r=1 / \operatorname{sqrt}(2)$.

Data Set 1. All ANOVA model comparisons are shown in Table 1. Note that because the Bayes factors in Table 1 all have the same denominator, the reader can divide any two Bayes factors of any two models to compute the respective Bayes factor. The winning model included Emphasis and Response main effects but no interaction between the two, $B F_{30}=1.168 \times 10^{14}$. The mean RT was faster for error responses 
than for correct responses regardless of the emphasis condition (see Figure 1).

Table 1

Model Comparison

\begin{tabular}{lrrrrr}
\hline Models & $\mathrm{P}(\mathrm{M})$ & $\mathrm{P}(\mathrm{M} \mid$ data $)$ & $\mathrm{BF}_{M}$ & $\mathrm{BF}_{10}$ & error \% \\
\hline Emphasis + Response & 0.200 & 0.614 & 6.371 & $1.168 \mathrm{e}+14$ & 1.467 \\
Emphasis + Response + Emphasis ${ }^{*}$ Response & 0.200 & 0.386 & 2.510 & $7.327 \mathrm{e}+13$ & 2.617 \\
Emphasis & 0.200 & $1.287 \mathrm{e}-4$ & $5.148 \mathrm{e}-4$ & $2.446 \mathrm{e}+10$ & 0.989 \\
Response & 0.200 & $3.024 \mathrm{e}-13$ & $1.210 \mathrm{e}-12$ & 57.472 & 1.079 \\
Null model & 0.200 & $5.262 \mathrm{e}-15$ & $2.105 \mathrm{e}-14$ & 1.000 & \\
\hline
\end{tabular}

Note. All models include subject

Because we did not find evidence for an interaction between Emphasis and Response, we did not conduct a simple effects analysis or estimate the related posterior distribution of the standardized effect size $\delta$. Overall at the group-level, we unexpectedly did not find evidence for slow error RTs in the archival data from Rae et al. (2014). We found evidence for fast error RTs, but the speed-accuracy emphasis manipulation did not produce the expected cross over effect, where speed emphasis produces fast errors and accuracy emphasis produces slow errors relative to correct responses. 


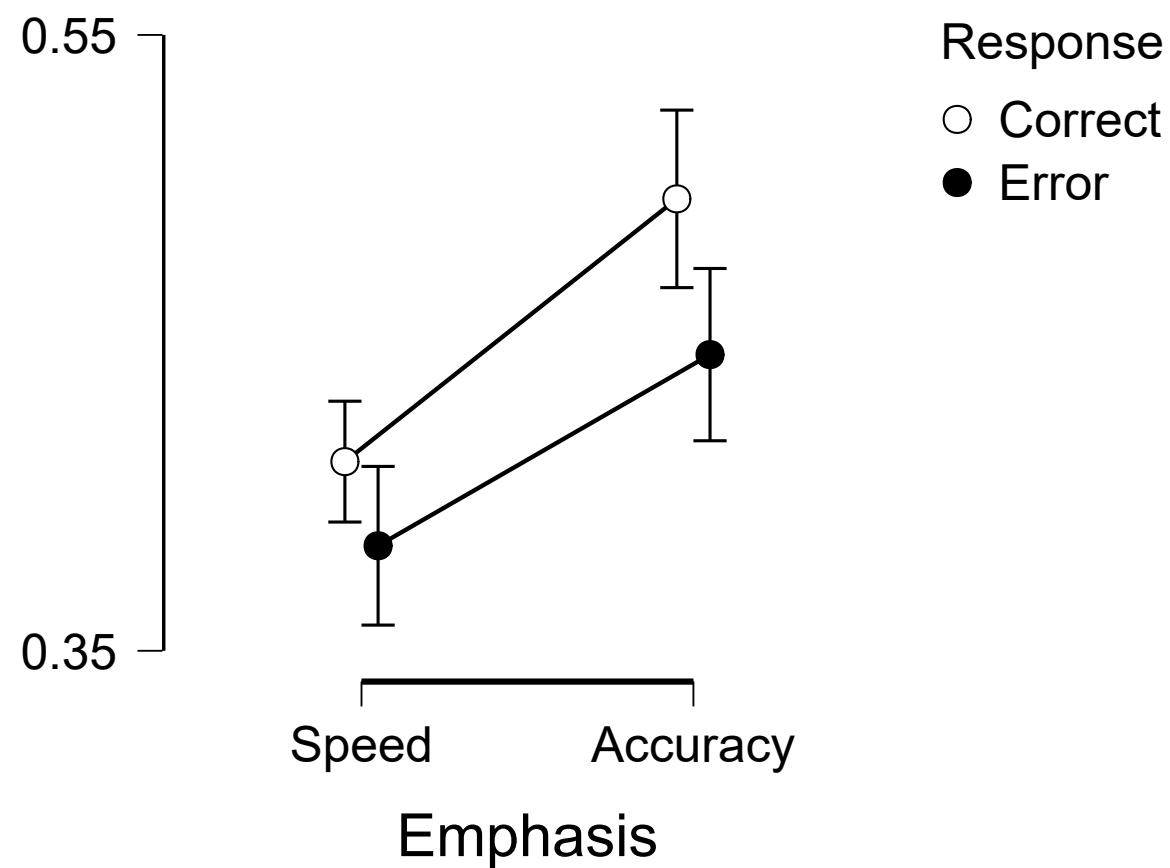

Figure 1. For data set 1, the mean response time for correct and error responses as a function of speed and accuracy emphasis. Error bars represent 95\% credible intervals.

Data Set 2. All ANOVA model comparisons are shown in Table 2. The winning model included Emphasis, Response, and the interaction between the two, $B F_{10}=3.321 \times 10^{39}$. Given the interaction, the differences between correct and error mean RT was explored via a simple effects analysis (see Figure 2). We found strong evidence for the hypothesis that there is a difference between correct and error mean RT in the speed condition, $B F_{10}=22.135$. But unexpectedly the mean RT for the error response was slower than the correct response. We found strong evidence for the hypothesis that there is a difference between correct and error RT in the accuracy condition, $B F_{10}=3.261 \times 10^{11}$. The mean RT was slower for the error response than the correct response. In regards to parameter estimation, in the speed condition, the median of the resulting posterior distribution for $\delta$ equals -.470 with a central $95 \%$ credible interval that ranges from -.772 to -.174. In the accuracy condition, the median of the resulting posterior distribution for $\delta$ equals -1.545 with a central $95 \%$ credible 
interval that ranges from -1.957 to -1.029 . Based on the effect sizes, it appears the speed manipulation does not necessarily produce absolute fast errors relative to correct responses, where the error meant RT is faster than the correct mean RT. But the speed emphasis manipulation can produce relatively faster errors compared to the accuracy condition. For instance, although both errors are absolutely slow errors (mean error RT slower than mean correct RT), in the speed condition, the magnitude of the slow error is much smaller compared to the accuracy condition.

Table 2

Model Comparison

\begin{tabular}{lrrrrr}
\hline Models & $\mathrm{P}(\mathrm{M})$ & $\mathrm{P}(\mathrm{M} \mid$ data $)$ & $\mathrm{BF}_{M}$ & $\mathrm{BF}_{10}$ & error \% \\
\hline Emphasis + Response + Emphasis * Response & 0.200 & 1.000 & 14204.576 & $3.321 \mathrm{e}+39$ & 1.648 \\
Emphasis + Response & 0.200 & $2.815 \mathrm{e}-4$ & 0.001 & $9.352 \mathrm{e}+35$ & 1.571 \\
Emphasis & 0.200 & $2.230 \mathrm{e}-9$ & $8.921 \mathrm{e}-9$ & $7.409 \mathrm{e}+30$ & 2.998 \\
Response & 0.200 & $5.589 \mathrm{e}-39$ & $2.236 \mathrm{e}-38$ & 18.568 & 1.097 \\
Null model & 0.200 & $3.010 \mathrm{e}-40$ & $1.204 \mathrm{e}-39$ & 1.000 & \\
\hline
\end{tabular}

Note. All models include subject

At the group-level, we unexpectedly did not find evidence for fast error RTs in the archival data from Evans, Rae, et al. (2017). This is in contrast to the Data Set 1 analysis where we did not find evidence for slow errors. Again, the speed-accuracy emphasis manipulation did not produce the expected cross over effect, where speed emphasis produces fast errors and accuracy emphasis produces slow errors relative to correct responses. From our parameter estimation we found that the slow errors were smaller in magnitude in the speed condition compared the accuracy condition, but there was almost no probability density for $\delta$ values greater than 0 (i.e., fast errors) in the speed condition. 


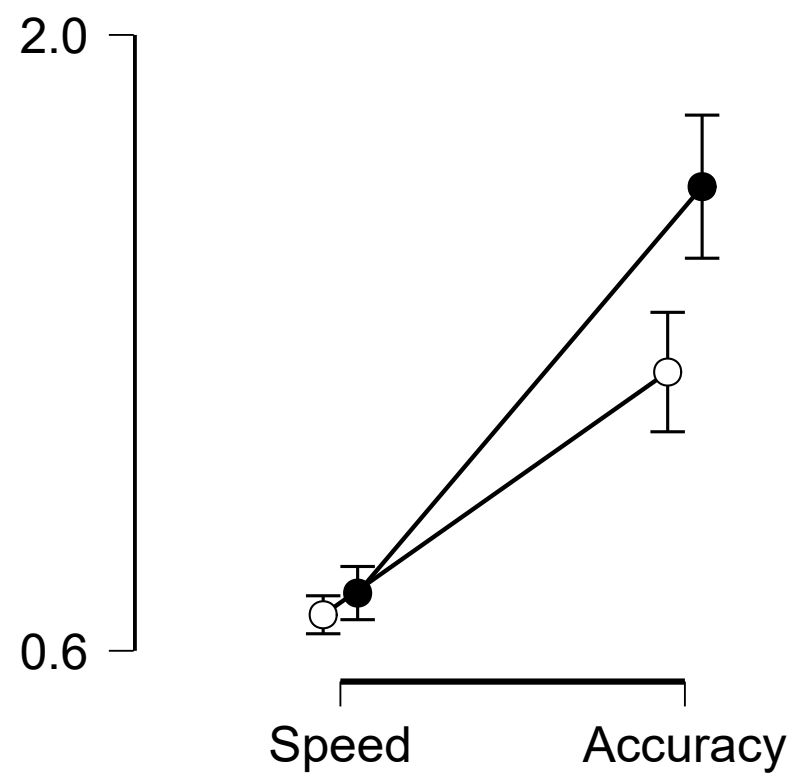

Response

O Correct

- Error

\section{Emphasis}

Figure 2. For data set 2, the mean response time for correct and error responses as a function of speed and accuracy emphasis. Error bars represent 95\% credible intervals.

Data Set 3. All ANOVA model comparisons are shown in Table 3. The winning model included Emphasis, Response, and the interaction between the two, $B F_{10}=6.625 \times 10^{75}$. The cross over interaction, see Figure 3, was explored via simple effects analysis. We found strong evidence for the hypothesis that there is a difference between Correct and Error mean RT in the Speed condition, $B F_{10}=9.797 \times 10^{31}$. The mean RT was faster for the error response. We found strong evidence for the hypothesis that there is a difference between Correct and Error RT in the Accuracy condition, $B F_{10}=1.039 \times 10^{5}$. The mean RT was slower for the error response.

Table 3

Model Comparison

\begin{tabular}{lrrrrr}
\hline Models & $\mathrm{P}(\mathrm{M})$ & $\mathrm{P}(\mathrm{M} \mid$ data $)$ & $\mathrm{BF}_{M}$ & $\mathrm{BF}_{10}$ & error \% \\
\hline Emphasis + Response + Emphasis * Response & 0.200 & 1.000 & $3.171 \mathrm{e}+7$ & $6.625 \mathrm{e}+75$ & 2.612 \\
Emphasis & 0.200 & $1.063 \mathrm{e}-7$ & $4.254 \mathrm{e}-7$ & $7.045 \mathrm{e}+68$ & 1.016 \\
Emphasis + Response & 0.200 & $1.980 \mathrm{e}-8$ & $7.919 \mathrm{e}-8$ & $1.312 \mathrm{e}+68$ & 2.255 \\
Response & 0.200 & $2.141 \mathrm{e}-77$ & $8.563 \mathrm{e}-77$ & 0.142 & 6.264 \\
Null model & 0.200 & $1.510 \mathrm{e}-76$ & $6.038 \mathrm{e}-76$ & 1.000 & \\
\hline
\end{tabular}

Note. All models include subject 
In regards to parameter estimation, in the speed condition, the median of the resulting posterior distribution for $\delta$ equals 1.905 with a central $95 \%$ credible interval that ranges from 1.623 to 2.218 . In the accuracy condition, the median of the resulting posterior distribution for $\delta$ equals -.559 with a central $95 \%$ credible interval that ranges from -.769 to -.351. At the group-level, our analyses of data set 3 support the notion that fast and slow error RT effects exist and that there is reasonable precision that these effects are large. Moreover, the sign of $\delta$ in the speed and accuracy conditions suggests that the speed-accuracy emphasis manipulation produces the expected cross over effect, where speed emphasis produces fast errors and accuracy emphasis produces slow errors relative to correct responses.

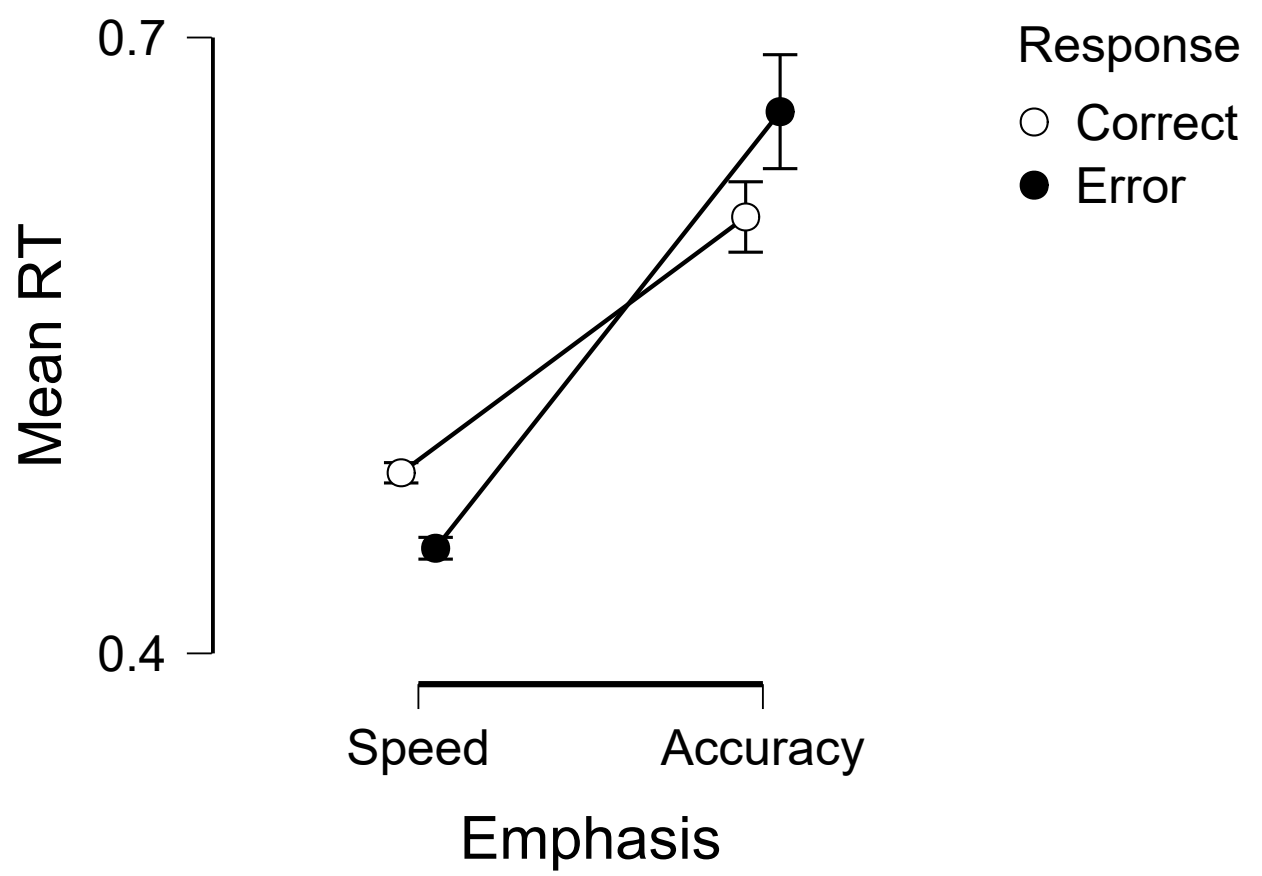

Figure 3. For data set 3, the mean response time for correct and error responses as a function of speed and accuracy emphasis. Error bars represent 95\% credible intervals.

\section{Interim Conclusions}

The group-level analyses demonstrated that the speed-accuracy trade-off manipulation did not reliably produce cross-overs between fast and slow errors due to 
speed-accuracy emphasis manipulations such as those reported by Ratcliff and Rouder (1998). All of the experiments examined here produced conflicting results with either all fast errors (i.e., Rae et al., 2014), all slow errors (i.e., Evans, Rae, et al., 2017), or the expected cross over effect found in the newly collected data set. Overall, fast and slow error effects may not be as reliable as commonly believed. Interestingly, there were no fast errors in Data Set 2 but the relative speed of the errors were faster compared to the accuracy condition.

The group-level analyses used an aggregate approach that does not allow us to address the individual variation in the participants. For example, the differences between studies could be due to actual differences in the methodology. However, within each study participants received the same methodology, and therefore, if fast and slow errors are ubiquitous, then they should at least show within-study consistency across participants. We turned to methods developed by Haaf and Rouder (2017) to investigate if all participants show fast/slow errors, if all participants show no effect, or if there are individual differences across participants within each condition of each experiment (e.g., some participants with fast errors and some with slow errors in the speed condition).

\section{Subject Level Analyses}

For the subject-level analyses we used the same three data sets as the group-level analyses. For each study, the speed and accuracy conditions were modelled as separate data sets, which we will refer to as sub-data sets. Within each of these sub-data sets there are two conditions: the correct responses and the error responses. The dependent variable we are interested in is the response time (RT). The general subject-level analysis we used here is described in detail in Haaf and Rouder (2017) and Haaf and Rouder (2019), but we will provide our study specific details here. In general, each 
sub-data set is modelled as

$$
Y_{i j k} \sim \operatorname{Normal}\left(\mu+\alpha_{i}+x_{j} \theta_{i}, \sigma^{2}\right)
$$

where $Y_{i j k}$ is the dependent variable RT, for the $k$ th trial, the $i$ th participant, and the $j$ th condition. The $\mu+\alpha_{i}$ term represents the intercepts where $\mu$ is the grand mean of intercepts and $\alpha_{i}$ is the deviations across participants. The term $x_{j}$ represents the conditions of the sub-data set, with $x_{j}=0$ for the error responses and $x_{j}=1$ for the correct responses. The $\theta_{i}$ parameter represents the effect size (i.e., difference between mean correct RT and mean error RT) slope for each participant $i$. The $\sigma^{2}$ parameter is the variance of the dependent variable. We test five different variations of this linear model, where we modify the model structure of the effect parameter $\theta_{i}$.

The Fast and Slow Error model is denoted $\mathcal{M}_{F S}$ and places no order constraints on the individual effects. This means that each participant can have a fast or slow error effect regardless of the condition. Recall that we expect speed emphasis to produce fast errors and accuracy emphasis to produce slow errors. The formal model can be expressed by the following equation:

$$
\mathcal{M}_{F S}: \quad \theta_{i} \sim \operatorname{Normal}\left(\nu, \eta^{2}\right),
$$

where $\nu$ and $\eta^{2}$ are population-level parameters describing the mean and variance of effects across the population. $\mathcal{M}_{F S}$ allows for both fast and slow errors in either the speed or accuracy conditions.

The Fast Error model, denoted $\mathcal{M}_{F}$, captures the constraint that each individual has only fast error effects. The formal model can be expressed by the following equation: 


$$
\mathcal{M}_{F}: \quad \theta_{i} \sim \operatorname{Normal}_{+}\left(\nu, \eta^{2}\right),
$$

where $\mathrm{Normal}_{+}$denotes a truncated normal distribution with a lower bound of zero. The Slow Error model, denoted $\mathcal{M}_{S}$, captures the constraint that each individual has only slow error effects. The formal model can be expressed by the following equation:

$$
\mathcal{M}_{S}: \quad \theta_{i} \sim \text { Normal }_{-}\left(\nu, \eta^{2}\right)
$$

where Normal_ denotes a truncated normal distribution with an upper bound at zero. The common-effect model, denoted $\mathcal{M}_{1}$, captures the constraint that each individual has the same effect size:

$$
\mathcal{M}_{1}: \quad \theta_{i}=\nu
$$

This common-effect model allows for the effects to be fast errors or slow errors, but assumes a constant effect across all individuals. If individual effects vary only a very small amount then $\mathcal{M}_{1}$ may be the winning model. The final model is the null model, denoted $\mathcal{M}_{0}$. The null model posits that each participant's true effect is exactly zero:

$$
\mathcal{M}_{0}: \quad \theta_{i}=0
$$

We analyze the above five models in a Bayesian framework similar to Haaf and Rouder (2017) and Haaf and Rouder (2019). $\mu$ and $\sigma^{2}$, which are common to all models, have the follow non-informative priors: 


$$
f\left(\mu, \sigma^{2}\right) \propto \frac{1}{\sigma^{2}}
$$

We use the same $g$-prior structure as Haaf and Rouder (2017) on the population effect parameter $\nu$, each individual's intercept $\alpha_{i}$, and each individual's effect parameter $\theta_{i}$. Under the $g$-prior specification, the following priors were placed on the $g$ parameter:

$$
g_{\alpha} \sim \text { Inverse- } \chi^{2}\left(r^{2}\right)
$$

where we set $r_{\alpha}^{2}=1.0, r_{\nu}^{2}=(1 / 6)^{2}$, and $r_{\theta}^{2}=(1 / 10)^{2}$.

Posterior distributions for all parameters in the $\mathcal{M}_{F S}$ and $\mathcal{M}_{1}$ models were sampled with Markov chain Monte Carlo methods (MCMC) using the BayesFactor package in R (Morey, Rouder, \& Jamil, 2014). We further inspect the posterior means of the $\theta_{i}$ parameters, which are shown in Figure 4 . The Bayesian estimates in data set 2 and 3 show large amounts of shrinkage suggesting a small amount of variation at the individual level, and therefore, the majority of the observed variation is present at the trial level. Based on these estimates we can conclude most participants show fast errors in Data Set 1 and slow errors in Data Set 2, regardless of the speed/accuracy condition. In Data Set 3, most participants showed fast errors in the speed condition and a small proportion showed fast errors in the accuracy condition.

To compare the five models, we use Bayes factors. The values for all 6 sub-data sets are provided in Table 4. The asterisk in each column marks the preferred model for each data set. The values in the table are the Bayes factor between the respective model and the preferred model. For example, for Data Set 1, the $\mathcal{M}_{F S}$ model is the preferred model. The table shows how much worse the other models perform compared 
to the preferred one. The preferred model for 4 out of the 6 sub-data sets suggest that participants have a mixture of fast and slow error effects in both the speed and accuracy conditions. Combined with the estimation results, we can infer that the proportion of participants in the same study that shows the unexpected effect (i.e., fast errors in the accuracy condition) is a small proportion of the total sample. In Data Set 2, all participants showed slow errors in the accuracy condition and in Data Set 3 all participants showed fast errors in the speed condition.

Table 4

Subject-Level Bayes factors for Model Comparison

\begin{tabular}{lllllll}
\hline & Rae Speed & Rae Accuracy & Evans Speed & Evans Accuracy & Tillman Speed & Tillman Accuracy \\
\hline $\mathcal{M}_{0}$ & 1 to $10^{133}$ & 1 to $10^{228}$ & 1 to $10^{7}$ & 1 to $10^{67}$ & 1 to $10^{169}$ & 1 to $10^{51}$ \\
$\mathcal{M}_{1}$ & 1 to $10^{26}$ & 1 to $10^{71}$ & 1 to $10^{3}$ & 1 to 5140 & 1 to $10^{7}$ & 1 to $10^{27}$ \\
$\mathcal{M}_{F}$ & 1 to $10^{2}$ & 1 to $10^{2}$ & 1 to $10^{2}$ & 1 to $10^{3}$ & $*$ & 1 to 7368 \\
$\mathcal{M}_{S}$ & 1 to $10^{2}$ & 1 to $10^{2}$ & 1 to 929 & $*$ & 1 to $10^{3}$ & 1 to 7521 \\
$\mathcal{M}_{F S}$ & $*$ & $*$ & $*$ & 1 to 9 & 1 to 11 & $*$ \\
\hline
\end{tabular}

Note. The ${ }^{*}$ indicates the winning model

\section{General Discussion}

We aimed to provide a more definitive assessment of two key qualitative benchmarks in speeded decision making: fast and slow error response times (RT). We re-analysed two archival data sets and one new data set that involved brightness discriminations under both speed and accuracy emphasis. The analyses we conducted determined if the effects exist at the group level and are consistent at the individual subject level. Overall, we found inconsistent patterns across experiments looking at group-level trends and inconsistent patterns across people looking at subject level trends. Our results suggest that fast and slow errors are not ubiquitous patterns of data across studies, but more importantly, these effects show strong individual differences across people who experience identical experimental conditions.

Group-level inconsistencies have previously been found in fast and slow patterns 
of errors, but not in the context that the results questioned the status of fast or slow errors as a benchmark. For instance, fast errors are not commonly observed in recognition memory experiments (Osth, Bora, Dennis, \& Heathcote, 2017; Ratcliff \& Smith, 2004). Our study is the first to highlight the inconsistencies in both fast and slow errors at group and subject levels and question whether these trends are qualitative benchmarks. Furthermore, our study is the first to hold fast and slow errors to the much higher theoretical standard of individual difference analyses, assessing whether everyone shows these patterns of data (Haaf \& Rouder, 2017, 2019).

Despite the inconsistencies we found in our study, we still observed errors that were not the same speed as correct responses. Table 4 shows that $\mathcal{M}_{0}$ was never the preferred model and this model represented the scenario where fast and slow errors do not exist. This means that it is still reasonable to suggest that fast and slow errors can occur, but we need to be cautious about assuming these effects are always present in data or consistent across individuals in the same data set. In relation to theory, this means that we need to be cautious about assuming that between-trial variability in drift rate and starting point - which were the additional mechanisms added to models such as the diffusion model (e.g., Ratcliff \& Rouder, 1998) to explain fast and slow errors - are necessary in all speeded decision-making contexts, as there may not be adequate justification for these mechanisms in many situations.

In a broader sense, we believe that our study necessitates a field-wide discussion on what exactly makes an effect a "qualitative benchmark", and what standards should a pattern of data have to meet to become a benchmark that we base theories and models on. For instance, the Stroop effect (see Stroop, 1935; Tillman, Eidels, \& Finkbeiner, 2016; Tillman, Howard, Garret, \& Eidels, 2017) has been replicated at the 
group level for the last 80 years (MacLeod, 1991) and is also consistent across individuals (Haaf \& Rouder, 2017, 2019), suggesting that it is a strong qualitative benchmark for developing theories. In the speeded decision making literature, a reliable effect is the positive skew of RT distributions, which is generally considered to be an ubiquitous trend (Evans, Hawkins, Boehm, Wagenmakers, \& Brown, 2017). While specific manipulations can be introduced to eliminate the positive skew (Evans \& Hawkins, 2019; Evans, Hawkins, \& Brown, 2019), which may lead some to claim that is has similar issues to those that we point out with fast and slow errors. However, the fact that we can directly control whether or not a positive skew occurs is a desirable finding in relation to qualitative benchmarks. This is because we have identified a ubiquitous occurrence with consistent boundary conditions that we can theorise about. Importantly, based on the results of our study, the patterns of fast and slow errors are highly inconsistent across experiments and participants, bringing into question their status as a qualitative benchmark for speeded decision making.

As a field, we believe that we should think more carefully about what requirements a pattern of data should need to meet to become a "benchmark". At minimum, we suggest that a qualitative benchmark that informs our theories and models should be a pattern of data that is reliable across experiments in the same context and people within the same study, and any deviations in the benchmark effect should be directly under the control of the experimenter in the form of manipulations or design. These high standards ensure that our understanding of cognitive processes are heavily informed by only the most reliable patterns of data, preventing us from building general theories of cognitive processes based on phenomena that inconsistently occur in limited cases. 

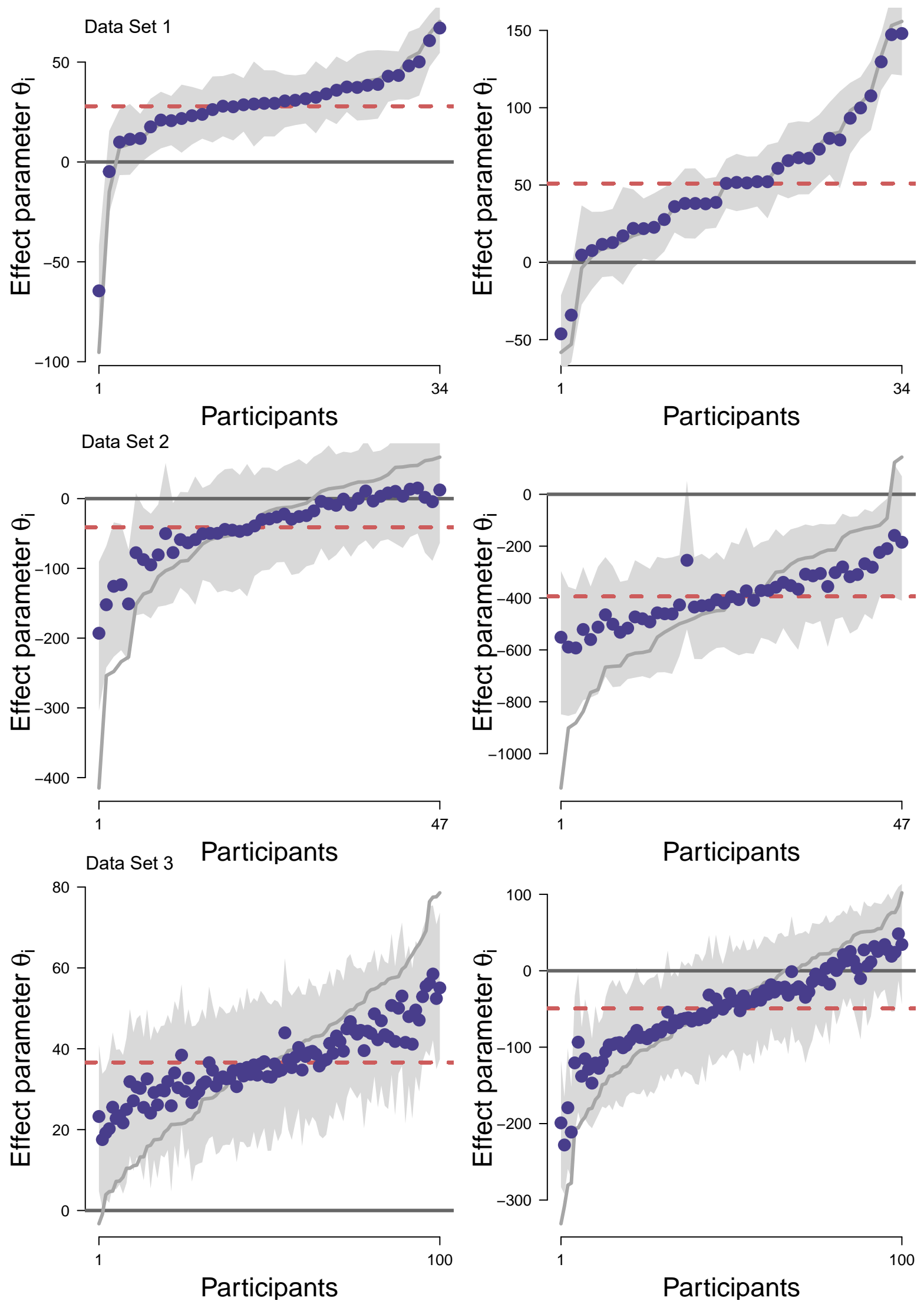

Figure 4. Empirical and Bayesian analyses for each data set, where each row shows one data set. The left column shows the ordered Bayesian estimates of individual effects for the speed condition and the right column shows the same for the accuracy condition. The blue dots are the estimates from the $\mathcal{M}_{F S}$ model and the red line is the effect estimate from the $\mathcal{M}_{1}$ model. The shaded area denotes the associated $95 \%$ credible intervals around the estimates of the $\mathcal{M}_{F S}$ model. The observered effects are included as a grey line for comparison. The difference between the observed and estimated effects represents hierarchical shrinkage. 


\section{References}

Brown, S. D., \& Heathcote, A. (2008). The simplest complete model of choice response time: linear ballistic accumulation. Cognitive psychology, 57(3), 153-178.

Donders, F. C. (1868). Die schnelligkeit psychischer processe: Erster artikel. Archiv für Anatomie, Physiologie und wissenschaftliche Medicin, 657-681.

Donkin, C., \& Brown, S. D. (2018). Response times and decision making. In T. Wixted \& E.-J. Wagenmakers (Eds.), The stevens' handbook of experimental psychology and cognitive neuroscience (4th ed., Vol. 5). New York: John Wiley \& Sons, Inc.

Evans, N. J., \& Hawkins, G. E. (2019). When humans behave like monkeys: Feedback delays and extensive practice increase the efficiency of speeded decisions. Cognition, 184, 11-18.

Evans, N. J., Hawkins, G. E., Boehm, U., Wagenmakers, E.-J., \& Brown, S. D. (2017). The computations that support simple decision-making: A comparison between the diffusion and urgency-gating models. Scientific reports, 7(1), 1-13.

Evans, N. J., Hawkins, G. E., \& Brown, S. D. (2019). The role of passing time in decision-making. Journal of experimental psychology: learning, memory, and cognition.

Evans, N. J., Rae, B., Bushmakin, M., Rubin, M., \& Brown, S. D. (2017). Need for closure is associated with urgency in perceptual decision-making. Memory \& cognition, 45(7), 1193-1205.

Haaf, J. M., \& Rouder, J. N. (2017). Developing constraint in bayesian mixed models. Psychological methods, 22(4), 779.

Haaf, J. M., \& Rouder, J. N. (2019). Some do and some dont? accounting for variability of individual difference structures. Psychonomic bulletin \& review, 26(3), 772-789.

Heitz, R. P. (2014). The speed-accuracy tradeoff: history, physiology, methodology, and behavior. Frontiers in neuroscience, $8,150$.

Kruglanski, A. W., Atash, M., De Grada, E., Mannetti, L., \& Pierro, A. (2013). Need for closure scale (nfc). measurement instrument database for the social science.

Laming, D. R. J. (1968). Information theory of choice-reaction times. London: Academic Press.

Luce, R. D. (1986). Response times: Their role in inferring elementary mental organization (No. 8). Oxford: Oxford University Press.

MacLeod, C. (1991). Half a century of research on the stroop effect: An integrative review. 
Psychological Bulletin, 109, 163-203.

Morey, R., Rouder, J., \& Jamil, T. (2014). Bayesfactor: Computation of bayes factors for common designs. $R$ package version $0.9,8$.

Osth, A. F., Bora, B., Dennis, S., \& Heathcote, A. (2017). Diffusion vs. linear ballistic accumulation: Different models, different conclusions about the slope of the zroc in recognition memory. Journal of Memory and Language, 96, 36-61.

Peirce, J. W. (2007). Psychopy-psychophysics software in python. Journal of Neuroscience Methods, 162(1), 8-13.

Rae, B., Heathcote, A., Donkin, C., Averell, L., \& Brown, S. (2014). The hare and the tortoise: Emphasizing speed can change the evidence used to make decisions. Journal of Experimental Psychology: Learning, Memory and Cognition, 40(5), 1226-43.

Ratcliff, R. (1978). A theory of memory retrieval. Psychological Review, 85(2), 59-108.

Ratcliff, R. (1993). Methods for dealing with reaction time outliers. Psychological bulletin, 114(3), 510.

Ratcliff, R. (2002). A diffusion model account of response time and accuracy in a brightness discrimination task: Fitting real data and failing to fit fake but plausible data. Psychonomic Bulletin \& Review, 9(2), 278-291.

Ratcliff, R., \& Rouder, J. N. (1998). Modeling response times for two-choice decisions. Psychological Science, 9(5), 347-356.

Ratcliff, R., \& Smith, P. L. (2004). A comparison of sequential sampling models for two-choice reaction time. Psychological review, 111(2), 333-67.

Ratcliff, R., Van Zandt, T., \& McKoon, G. (1999). Connectionist and diffusion models of reaction time. Psychological review, 106(2), 261.

Smith, P. L., \& Vickers, D. (1988). The accumulator model of two-choice discrimination. Journal of Mathematical Psychology, 32(2), 135-168.

Stone, M. (1960). Models for choice-reaction time. Psychometrika, 25 (3), 251-260.

Stroop, J. R. (1935). Studies of interference in serial verbal reactions. Journal of Experimental Psychology, 18(6), 643-662.

Swensson, R. G. (1972). The elusive tradeoff: Speed vs accuracy in visual discrimination tasks.

Perception \&3 Psychophysics, 12(1), 16-32. 
The JASP Team. (2019). Jasp (version 0.9.2)[computer software]. https://jasp-stats.org/.

Tillman, G., Eidels, A., \& Finkbeiner, M. (2016). A reach-to-touch investigation on the nature of reading in the stroop task. Attention, Perception, 83 Psychophysics, 78(8), 1 - 11.

Tillman, G., Howard, Z., Garret, P., \& Eidels, A. (2017). The stroop effect from a mixture of reading processes: A fixed-point analysis. Proceedings of the 39th Annual Conference of the Cognitive Science Society.

Tillman, G., Van Zandt, T., \& Logan, G. D. (2020). Sequential sampling models without random between-trial variability: The racing diffusion model of speeded decision making. Psychonomic Bulletin \& Review.

Usher, M., \& McClelland, J. L. (2001). The time course of perceptual choice: The leaky competing accumulator model. Psychological Review, 108, 550-592.

Wickelgren, W. A. (1977). Speed-accuracy tradeoff and information processing dynamics. Acta psychologica, 41(1), 67-85. 